\title{
Methods to Address Computed Tomography-Related Risk Factors in Oncology Patients: An Expert Opinion Based on Current Evidence
}

\author{
Lucia Del Mastro $^{\text {a }}$ Andrea Laghi $^{\text {b Claudio Ronco }}{ }^{c}$ \\ ${ }^{a}$ Department of Internal Medicine, University of Genova - Ospedale Policlinico San Martino, Genova, Italy; \\ bepartment of Radiological Sciences, Oncology and Pathology, "Sapienza" University of Rome, AOU Sant'Andrea, \\ Rome, Italy; ${ }^{\mathrm{C} D e p a r t m e n t ~ N e p h r o l o g y}$ Dialysis and Transplantation, International Renal Research Institute (IRRIV), \\ Vicenza, Italy
}

\section{Keywords}

Multidetector computed tomography - Oncology patients . Contrast media - Radiation exposure - Risk assessment .

Acute kidney injury

\begin{abstract}
Background: The present paper is intended to be a practical guide organized by statements describing methods to reduce risks related to $C T$ examinations in cancer patients. Methods: A panel of radiologists, oncologists and nephrologists were selected based on their publication records in the field and expertise. Ten clinical questions, which were derived from clinical needs and an integration of all the committee members' suggestions, were stated. The modified Delphi approach was used; it involved a detailed literature review and the collective judgement of experts, including electronic and face-to-face discussions. Results: Ten statements were derived from expert opinions based on the current literature, recently developed guidelines and technological advancements. Each statement is discussed in a short paragraph re-
\end{abstract}

porting the current key evidence. Conclusion: This paper addresses the clinical implications of performing numerous CT examinations in patients with cancer, providing clinicians with information regarding methods to reduce risk factors in this patient population.

(c) 2018 The Author(s)

Published by S. Karger AG, Basel

\section{Background}

Cancer represents the second most important cause of death and morbidity in Europe, with more than 3.7 million new cases and 1.9 million deaths reported each year. In the United Kingdom, the number of cancer survivors has increased by $3 \%$ per annum [1]. Overseas, the American Cancer Society, in collaboration with the National Cancer Institute, estimates that more than 15.5 million cancer survivors are living in the United States today, and that number is expected to increase to 20 million by 2026 [2]. Consequently, over the next decade, the number of cancer survivors is expected to increase worldwide. 
The growing number of patients with cancer and cancer survivors results from several factors. First, advances in the scientific understanding and the precision of medical treatments have improved patient outcomes and quality of life. Second, improvements in early detection have simplified cancer treatment. Third, the ageing population may be the most influential factor affecting the increasing number of these patients.

Despite the variability related to cancer type, the median age of patients at the time of cancer diagnosis is 65 years old. Of the $47 \%$ of cancer survivors, almost half are 70 years old or older, and only $5 \%$ are younger than 40 [2]. An aging population will increase the number of patients whose cancer will be complicated by other chronic diseases. For these reasons, the management of patients with cancer will be particularly challenging, and a panel of specialists with different areas of expertise will play a crucial role.

Patients with cancer usually undergo several imaging examinations that generally require the intravascular administration of contrast medium (CM), particularly CT. One of the most subtle but relevant risks related to iodinated contrast agents is acute kidney injury (AKI), which occurs in $2.3 \%$ of all CT examinations. The risks of developing this complication are compounded by several patient conditions, such as advanced age, dehydration and coadministration of nephrotoxic chemotherapeutic drugs. Primary care physicians and all specialists involved in managing patients with cancer should have a basic understanding of these risks and should be aware of recent developments in CM and CT scanners that can potentially reduce them.

In this article, we review the definition, epidemiology, possible mechanisms and risk factors for contrast-induced AKI (CI-AKI) and provide recommendations for preventing this potentially life-threatening complication. Therefore, the aim of the present study is to provide a practical overview of the most common risk factors to be considered in patients with cancer before performing a contrast-enhanced CT (CECT) examination while providing an evidence-based approach to reduce cancer risks related to $\mathrm{CM}$ and radiation exposure during medical imaging.

\section{Methods}

A panel of radiologists, oncologists and nephrologists chaired by A.L., C.R., and L.D.M. were selected based on their publication records in the field, expertise and geographical location to ensure an appropriate representation across the country. Research fellows were considered for an appointment to the committee to help with some of the tasks, such as researching published studies and drafting documents. All the invited members are listed as contributors. Demographics of all experts involved are reported in online supplementary Appendix 1 (see www.karger.com/ doi/10.1159/000486821). The specific role of all members in this project is reported in the Author Contribution Section.

A modified Delphi approach was used; it involved a detailed literature review and the collective judgement of experts, including electronic and face-to-face discussions. Under the direction of the coordinating team (D.B., A.L., L.D.M., and C.R.), the committee produced the initial questions containing all items for which a consensus statement is planned. The draft questionnaire was sent to all members and modified based on feedback from all committee members. The coordinating team convened 3 working groups, one composed of radiologists, 1 group of nephrologists and 1 group of oncologists, and allocated the key questions to these task forces according to the subspecialities of the members involved.

Each working group performed a systematic literature search to prepare evidence-based statements for their assigned key questions. Medline, EMBASE and other databases were searched, including the following minimum search terms: computed tomography, oncology patients, CT acquisition protocol, contrast enhancement, low-dose CT scanner, contrast media osmolality, CI-AKI, renal function, biomarkers, radiation exposure and risk evaluation.

All articles investigating risks correlated to CM and radiation exposure in patients affected by any cancer were selected by inspecting the title and abstract. Articles reporting risks related to gadolinium-based contrast media were excluded. After further exploring the content, each task force summarized the included articles in a table of evidence. The final search results were circulated to all committee members, along with full abstracts and full papers. Working groups were free to update the literature search at their discretion. Studies published up to November 2016 were included.

Each task force prepared statements answering their assigned key questions. The statements were subsequently discussed and voted on during a face-to-face meeting of the entire group held on November 9, 2016. In January, 2017, a draft prepared by the coordinating team (D.B., A.L., L.D.M., and C.R.) was sent to all group members for comments. After definitive approval of the final draft, the language of the manuscript was reviewed and then submitted to the journal. Due to the practical intent of the current study and the lack of specific relevant evidence for these topics, no specific grading system was used.

\section{Results}

Clinical questions, which were derived from clinical needs and an integration of all the committee members' suggestions, are stated below. The statements and recommendations appear in italics.

\section{Is There an Increased Risk of AKI in Patients with}

Cancer?

The incidence of AKI in patients with cancer ranges from 12 to $17 \%$, and it is higher than the incidence observed in patients without cancer (5-8\%). Risk factors include the type of cancer, comorbidities, co-treatment with FANS/fu- 
rosemide, antibiotics, chemotherapy and CM administration. Among drugs commonly used for chemotherapy and targeted therapy, an increased risk of AKI has been documented in patients treated with bevacizumab, pemetrexed, cetuximab, ifosfamide and the platinum derivative cisplatin. Specific cancer locations (esophagus, kidney, liver, pancreas, craniopharyngeal duct, heart/mediastinum and uterus) and cancer types (lymphoma, leukemia, mixed lymphosarcoma, multiple myeloma) correlate with an increased risk of AKI.

Should an Iodate CM Injection be Considered a

Relevant Risk Factor for AKI?

Iodate CM is a risk factor for AKI. The risk for AKI associated with $C M(O R$ 4.55) is higher than the risk observed for other risk factors, such as diabetes, chemotherapy, hyponatremia and antibiotic use. A short time span between chemotherapy administration and CM injection is associated with an increased risk of AKI.

Is the Use of Iso-Osmolar Contrast Media Preferable to the Use of Low Osmolar Contrast Media as a

Strategy to Prevent CI-AKI in Patients with Cancer?

The use of CM with the lowest osmolarity is advisable, particularly in high-risk patients. Patients with cancer must be considered as a high-risk group, and Iso-Osmolar Contrast Media (IOCM) should be considered the first choice, particularly if the patients are affected by at least one of the following conditions: intra-arterial injection, diabetes mellitus, liver diseases, hypertension, pre-existing CKD ( $\mathrm{SCr}$ levels $>2 \mathrm{mg} / \mathrm{dL}$ ), hematocrit $<30 \%$, age over 70 years, cardiac diseases, and recent myocardial infarction ( $<1$ month). Iodixanol is the first choice for patients with myeloma or patients with monoclonal gammopathies, independent of additional risk factors.

Is There Any Biological and/or Clinical Evidence That Supports the Differential Impact of IOCM and LOCM on Renal Toxicity in Patients with Cancer? Does CM Viscosity Have a Role in Renal Toxicity?

Consistent evidence from cultured tubular cells has indicated that LOCM has more severe cytotoxic effects than IOCM. In addition, in animal models, IOCM is associated with a lower induction of Nox4-dependent reactive oxygen species (ROS) generation. IOCM also exerts fewer vasoconstriction effects than LOCM.

The role of viscosity in CI-AKI risk is still being debated. All CM, which are more viscous than plasma, and agents with increased osmolality that have lower viscosity may still lead to AKI.
Should the Renal Function of all Patients with Cancer be Assessed before the Administration of CM?

Renal function (SCr levels or GFR) should be assessed in all patients with cancer before the administration of CM to define the best strategy to prevent AKI.

Is the Serum Creatinine Level the Only Clinical Criterion Used to Define AKI or CM-Induced Toxicity?

The combination of markers of functional damage (elevation in Serum Creatinine [SCr] levels) and structural damage (biomarkers) guarantees more accurate identification of patients with subclinical AKI or those at a higher risk of developing persistent kidney damage.

Does Radiation Exposure for Diagnostic Purposes

Increase the Risk of Developing New Forms of

Cancer in Patients with Pre-Existing Cancer?

The risk of malignancy from diagnostic radiation exposure in adults is generally low. The uncertainty surrounding this issue should prompt the use of low-dose protocols and adequate technology. Clinicians should take precautions with patients who have been exposed to $10 \mathrm{mSv}$ for a year because these patients have an increased risk of developing new forms of cancer.

How Should CT Protocols be Optimized for Oncology Patients?

CT protocols should be personalized according to the patient, tumor characteristics and needs to minimize radiation exposure and the amount of CM while maintaining adequate image quality. CT-specific protocols (number of phases acquired and body regions to be scanned), including the adoption of modern techniques for dose reduction (Automatic Dose Modulation and iterative reconstruction algorithms) should be implemented while considering tumour characteristics and different clinical settings, as well as the diagnostic work-up and disease staging, evaluation of the response to treatment and long-term follow-up.

How Should Contrast Enhancement be Optimized for Standard and Low-Dose Acquisition Protocols in Oncology Patients?

Adequate liver enhancement requires personalization of the amount of CM. The CM dose should be calculated according to $\mathrm{mgI}$ rather than volume. The CM dose should be defined according to total body weight $(T B W)$ and, in patients with a BMI $>25$, to lean body weight. A range of 500-600 $\mathrm{mgI}$ per $\mathrm{kg} \mathrm{BW}$ is considered
Del Mastro/Laghi/Ronco 
adequate for liver enhancement. Adequate enhancement of hypervascular liver lesions requires a minimum iodine delivery rate of $1.2 \mathrm{gI} / \mathrm{s}$ using a standard acquisition protocol.

In Patients with Cancer, Does the Use of a Dimeric

IOCM Permit Better Diagnostic Imaging in

Low-Dose Procedures than Standard Protocols?

No evidence supports the superiority of a specific CM in patients with cancer. The use of IOCM should be preferred due to its higher safety profile and patient tolerance, particularly in intra-arterial injections. Similar trends, although with weaker evidence, are observed for intravenous injections.

\section{Discussion}

The statements and recommendations appear in italics and are stated below. Each statement is discussed in a short paragraph reporting the current key evidence.

\section{Statement 1}

The incidence of AKI in patients with cancer ranges from 12 to $17 \%$, and it is higher than the incidence observed in patients without cancer (5-8\%). Risk factors include the type of cancer, comorbidities, co-treatment with FANS/furosemide, antibiotics, chemotherapy and CM administration. Among drugs commonly used for chemotherapy and targeted therapy, an increased risk of AKI has been documented in patients treated with bevacizumab, pemetrexed, cetuximab, ifosfamide and the platinum derivative cisplatin. Specific cancer locations (esophagus, kidney, liver, pancreas, craniopharyngeal duct, heart/mediastinum and uterus) and cancer types (lymphoma, leukemia, mixed lymphosarcoma, multiple myeloma) correlate with an increased risk of AKI.

AKI, which was formerly called acute renal failure, is commonly defined as an abrupt decrease in renal function and clinically manifests as a reversible acute increase in nitrogen waste products that is determined by measuring blood urea nitrogen and SCr levels over the course of hours to weeks (KDIGO; Table 1) [3].

Patients with cancer must be considered a population at high risk of developing AKI [4]. In a Danish population including 37,267 cancer patients, the risk of AKI (defined as a $>50 \%$ increase in SCr levels) was approximately $17.5 \%$ within 1 year of a cancer diagnosis and $27 \%$ after 5 years [5]. The rate of AKI in hospitalized patients with cancer seems to be higher than the rate re-

CT-Related Risk Factors in Oncology Patients
Table 1. CI-AKI definition. The 2007 guidelines of the AKIN defined CI-AKI as an absolute change in the SCr levels of $\geq 0.3 \mathrm{mg} /$ $\mathrm{dL}$ after an i.v. injection of CM

\begin{tabular}{lll}
\hline Stage & SCr levels & Urine output \\
\hline I & $\begin{array}{l}1.5-1.9 \times \text { baseline } \\
\text { Or increase of } \geq 0.3 \mathrm{mg} / \mathrm{dL} \\
(\geq 26.5 \mathrm{mmol} / \mathrm{L})\end{array}$ & $<0.5 \mathrm{~mL} / \mathrm{kg} / \mathrm{h}$ for $6-12 \mathrm{~h}$ \\
& $2.0-2.9 \times$ baseline & $<0.5 \mathrm{~mL} / \mathrm{kg} / \mathrm{h}$ for $\geq 12 \mathrm{~h}$ \\
\hline II $\quad$ & \\
\hline III $\quad$ & $3.0 \times$ baseline & \\
& Or increase to $\geq 4.0 \mathrm{mg} / \mathrm{dL}$ \\
& $(\geq 353.6 \mathrm{mmol} / \mathrm{L})$ \\
& Or initiation of RRT \\
& Or decrease in eGFR to $<35 \mathrm{~mL} /$ \\
& min $/ 1.73 \mathrm{~m}^{2}$ in patients $<18$ \\
& years old
\end{tabular}

AKIN, acute kidney injury network; KDIGO, kidney disease improving global outcomes; RRT, renal replacement therapy.

ported for hospitalized patients without cancer (12 vs. $5-8 \%$ respectively) $[4,6,7]$. Chertow et al. [7] evaluated the effects of AKI on the clinical outcomes and costs related to hospital stays for patients with cancer. They reported a 4.7 -fold increase in death, a 3-day increase in the length of the hospital stay, and a USD 42,671 increase in costs for hospitalized patients. Consequently, AKI has a relevant clinical and economic impact that must be considered.

Numerous factors and patient characteristics contribute to the increased risk of developing AKI in this patient population [8]. The OR for developing AKI is significantly higher for patients with diabetes (OR 1.89; 95\% CI $1.51-2.36$ ), those receiving chemotherapy (OR 1.61; 95\% CI 1.26-2.05), those given an intravenous injection of CM (OR 4.55; 95\% CI 3.51-5.89), patients with hyponatremia (OR 1.97; 95\% CI 1.57-2.47) and patients who use antibiotics (OR 1.52; 95\% CI 1.15-2.02) [4]. Heart failure, a recent myocardial infarction ( $<1$ month), hypertension, preexisting chronic kidney disease (CKD), and an age over 70 years must also be considered additional risk factors [9]. Moreover, patients with cancer originating in their oesophagus, kidney, liver, pancreas, craniopharyngeal duct, heart/mediastinum, or uterus and patients affected by lymphoma, leukaemia, mixed lymphosarcoma or multiple myeloma are considered a very high risk population with a $\geq 15 \%$ probability of developing AKI [4].

Medications play a critical role. According to the Renal Insufficiency and Anticancer Medications study [10], 
$50-60 \%$ of cancer patients who are undergoing antineoplastic treatment develop a renal impairment. Patients with cancer are exposed to multiple nephrotoxic agents (cytotoxic drugs, antibiotics, analgesics and drugs for supportive therapy). Both cytotoxic and cytostatic drugs are characterized by a specific nephrotoxic effect (Table 2). Ifosphamide and platinum agents (cisplatin and carboplatin) cause both glomerular and tubular damage. Cisplatin nephrotoxicity is the most common form of toxicity, occurring in $30-100 \%$ of children, and it typically causes glomerular impairment and tubulopathy, which clinically presents as magnesuria and hypomagnesemia [10]. The incidence and severity of renal toxicity increases with repeated usage of cisplatin-based chemotherapy, and CI-AKI may subsequently become irreversible. Thus, cisplatin discontinuation is generally indicated in patients who present signs of progressive renal impairment [11].

Some targeted agents may induce glomerular endothelial cell swelling, vacuolization and detachment, as well as disruption of epithelial cell slit diaphragms [12]. Cetuximab induces hypomagnesemia resulting from a renal magnesium leak. Bevacizumab induces hypertension and kidney-specific injury, including proteinuria and AKI.

\section{Statement 2}

Iodate CM is a risk factor for AKI. The risk for AKI associated with CM (OR 4.55) is higher than the risk observed for other risk factors, such as diabetes, chemotherapy, hyponatremia and antibiotic use. A short time span between chemotherapy administration and CM injection is associated with an increased risk of $A K I$.

Iodinated $\mathrm{CM}$ is frequently used for interventional and diagnostic procedures in patients with cancer. Intraarterial or intravenous injection of these agents increases patients' risks of developing CI-AKI. Salahudeen et al. [4] showed that in hospitalized cancer patients, the OR for developing AKI is significantly higher for intravenous contrast injections (OR 4.55). Several factors contribute to the increased risk, including the type and volume of $\mathrm{CM}$, frequency of contrast-enhanced imaging, comorbidities, underlying renal function and chemotherapies $[3,9,13-15]$. Hypertension and the combination of bevacizumab/irinotecan may be additional risk factors for CIAKI [8].

The effects of the administration of CM and chemotherapy within a short time have been widely investigated. Exposure to CM within a week before cisplatin administration increased the risk of AKI by 2.56-fold [11]. Cicin et al. [8] reported an incidence of CI-AKI
Table 2. Kidney injuries associated with chemotherapeutic agents. from Perazzella et al. [12]

Renal vasculature

Hemodynamic AKI (capillary leak syndrome)

IL-2, denileukin diftitox

Thrombotic microangiopathy

Antiangiogenesis drugs (bevacizumab and tyrosine kinase inhibitors)

Gemcitabine and cisplatin

Mitomycin C and IFN

Glomeruli

Minimal change disease

IFN

Pamidronate

Focal segmental glomerulosclerosis

IFN

Pamidronate

Zoledronate (rare)

Tubulointerstitium

Acute tubular necrosis

Platinums, zoledronate, ifosfamide, and mithramycin

Pentostatin, imatinib, diaziquone, and pemetrexed

Tubulopathies

Fanconi syndrome

Cisplatin, ifosfamide, and azacitadine, diaziquone, imatinib, and pemetrexed

Salt wasting

Cisplatin and azacitadine

Magnesium wasting

Cisplatin, cetuximab, and panitumumab

Nephrogenic diabetes insipidus

Cisplatin, ifosfamide, and pemetrexed

Syndrome of inappropriate antidiuresis

Cyclophosphamide and vincristine

Acute interstitial nephritis

Sorafenib and sunitinib

Crystal nephropathy

Methotrexate

IFN, interferon.

after CT in hospitalized oncology patients of $20 \%$. Moreover, CI-AKI developed 4.5 times more frequently in patients with cancer who had undergone a CT within 45 days after their last chemotherapy treatment $(p=0.005)$, which was also considered an independent risk factor $(p=0.017)$. In patients with cancer who require CECT in an emergency setting, the OR of developing CI-AKI (increase in SCr levels of $\geq 0.5 \mathrm{mg} / \mathrm{dL}$ or $\geq 25 \%$ within $48-72 \mathrm{~h}$ ) was 4.09 (95\% CI $1.34-12.56$ ) in patients requiring serial examinations on consecutive days compared with patients who only received scans on 1 day [16].
60

Blood Purif 2018;46:56-69 DOI: $10.1159 / 000486821$
Del Mastro/Laghi/Ronco 


\section{Statement 3}

The use of CM with the lowest osmolarity is advisable, particularly in high-risk patients. Patients with cancer must be considered as a high-risk group, and IOCM should be considered the first choice, particularly if the patients are affected by at least one of the following conditions: intra-arterial injection, diabetes mellitus, liver diseases, hypertension, pre-existing CKD ( $\mathrm{SCr}$ levels $>2 \mathrm{mg} / \mathrm{dL}$ ), hematocrit $<30 \%$, age over 70 years, cardiac diseases, and recent myocardial infarction $(<1$ month). Iodixanol is the first choice for patients with myeloma or patients with monoclonal gammopathies, independent of additional risk factors.

Evidence of the reduced incidence of CI-AKI in the general population and patients with cancer who have received IOCM has emerged from several studies, including systematic reviews and meta-analyses $[3,9,14]$. Different CM have been developed, each with their own pharmacological characteristics [17]. The most important feature for determining the risk of CI-AKI seems to be the osmolality of the CM, and the risk is higher for agents with increased osmolality compared to that of IOCM [18].

For example, with intra-arterial administration, the relative risk of AKI following iso-osmolar iodixanol, compared with the risk associated with a $\mathrm{CM}$ with increased osmolality, was 0.46 (95\% CI 0.27-0.79; $p=0.004$ ) using a CI-AKI definition based on absolute changes in SCr levels of $\geq 0.5 \mathrm{mg} / \mathrm{dL}$ [18]. As shown in the meta-analysis by Dong et al. [19] compared with LOCM, intra-arterial administration of iodixanol significantly decreased the risk of CI-AKI (risk ratio 0.68 ; $95 \%$ CI $0.50-0.92$; $\mathrm{Z}=$ $2.47 ; p=0.01)$ with no significant heterogeneity among studies $\left(p=0.14, \mathrm{I}^{2}=32.4 \%\right)$.

To date, a single randomized, prospective, comparative study of oncology patients with patients who have a very low risk of CI-AKI, which was defined as a glomerular filtration (GFR) rate $>60 \mathrm{~mL} / \mathrm{min}$, has been conducted. The results from an interim analysis showed a lower incidence of AKI in patients who have received an intravenous injection of IOCM $(0.5 \%)$ compared to iopromide (0.5 and $2.8 \%$ respectively) [20].

Intravenous administration of iodine-based contrast agent in patients with multiple myeloma complicated with monoclonal gammopathies is associated with a high risk of AKI. The risk is increased by comorbidities, such as CKD, hypercalcemia, diabetes, dehydration, and use of nephrotoxic drugs. According to the Preda study, the use of iodixanol appears to be safe in patients with monoclonal gammopathies and an eGFR $\geq 60 \mathrm{~mL} / \mathrm{min} / 1.73 \mathrm{~m}^{2}$

CT-Related Risk Factors in Oncology Patients and does not seem to be an absolute contraindication to the intravenous administration of iso-osmolar iodinebased contrast agent in patients with monoclonal gammopathies [21]. Concerning intravenous administration, the Nguyen study, which enrolled patients with decreased renal function, showed an inferior incidence of CI-AKI in patients treated with iodixanol (8.5\%) compared with the iopromide group $(27.8 \% ; p=0.012)$ [22]. Based on the Wang study, all-cause mortality was higher (1.5- to 1.8fold) for patients in the CKD subgroup who received LOCM than for patients who received IOCM, with a clear trend towards significance, and LOCM was associated with higher odds (approximately 1.6-fold) of a long-term elevation of SCr level than IOCM when adjusted for inverse probability weights [23].

Several guidelines recommend that contrast agents with the lowest osmolality should be used and high-risk patients should be administered IOCM, regardless of the administration route $[3,9,14]$. The guidelines of ACCF and AHA recommend the use of IOCM as a strategy to prevent AKI in patients with stable ischaemic heart disease [24]. Based on the Onco-Nephrology Curriculum of the American Society of Nephrology, high-osmolar (>1,400 mOsm/kg) and low osmolar (600-800 mOsm/ $\mathrm{kg}) \mathrm{CM}$ are associated with a higher incidence of AKI compared to IOCM (300 mOsm $/ \mathrm{kg})$. Preventive measures should be implemented in oncology patients with GFR $<60 \mathrm{~mL} / \mathrm{min}$, including limiting contrast volume and using IOCM.

\section{Statement 4}

Consistent evidence from cultured tubular cells has indicated that LOCM has more severe cytotoxic effects than IOCM. In addition, in animal models, IOCM is associated with a lower induction of Nox4- dependent reactive oxygen species (ROS) generation. IOCM also exerts fewer vasoconstriction effects than LOCM. The role of viscosity in CI-AKI risk is still being debated. All CM, which are more viscous than plasma, and agents with increased osmolality that have lower viscosity may still lead to AKI.

$\mathrm{CM}$ osmolality has been suggested to play a key role in determining renal toxicity, as documented by in vitro and in vivo models. LOCM have less severe nephrotoxic effects than high-osmolar ionic contrast media $[18,19]$. In addition, in high-risk patients, the iso-osmolar dimer iodixanol is associated with fewer nephrotoxic effects than LOCM [25]. The combination of both ischaemic and chemotoxic injury in the proximal tubules triggers a process called tubule glomerular feedback, which reduces filtration at the glomerulus level, increasing plasma concentra- 
tions of creatinine ( $\mathrm{Cr}$ ) approximately $24-48 \mathrm{~h}$ after a significant reduction in filtration [26]. Iodinated CM is a water-soluble and freely filtered agent that does not injure the glomerulus. Therefore, haematuria is not observed. However, its water solubility allows for relatively easy reabsorption by the proximal tubular cells, where it causes cellular damage [27]. Based on recent evidence, the mitochondria of damaged renal tubular cells release intracellular catalytic or unbound iron, which serves as the catalyst in the Haber-Weiss and Fenton reactions to drive oxidative stress and the production of the dangerous hydroxyl radicals [28]. The finding that LOCM exerts fewer nephrotoxic effects than high-osmolar ionic contrast media in vivo correlates with its reduced cytotoxic effects in vitro [29].

Netti et al. [30] investigated the effects of LOCM exposure on NADPH-dependent ROS generation by tubular cells. Iohexol, iopamidol and iodixanol were assessed at equimolar iodine concentrations, and their effects on human renal proximal tubular cells were studied/monitored/described (PTCs). Cytotoxicity, apoptosis and necrosis were investigated. At angiographic concentrations, iodixanol induces fewer cytotoxic effects on cultured tubular cells than iohexol and iopamidol and generates lower levels of Nox4-dependent ROS.

Other pharmacological characteristics of $\mathrm{CM}$, such as viscosity, which is mainly related to the size rather than the number of particles, are not believed to have a clinical impact on CI-AKI risk, although this is still under debate. Two main aspects should be considered. First, CM viscosity is related to temperature. As the temperature of a given $\mathrm{CM}$ increases, a concomitant decrease in its dynamic viscosity is observed. Therefore, warmed CM are less viscous than room temperature CM. Second, all CM are more viscous than plasma. According to $\mathrm{McCullough}$ et al. [25], contrast agents with increased osmolality that have lower viscosity may still lead to AKI. However, Seeliger et al. [31] suggested a relevant role for CM viscosity in the development of CI-AKI and that increased viscosity compromises blood flow and oxygen supply to critical regions of the kidney. The compromised flow delays the tubule glomerular feedback, probably resulting from increased tubular fluid viscosity, and impairs GFR. Although various properties of iodinated contrast agents, such as osmolality, ionic strength, viscosity and iodine content, have been implicated in renal toxicity, osmolality is the principal factor $[25,32]$.

Once CM has been administered, its osmolality is a not modifiable characteristic. Conversely, the viscosity may change due to thixotropy, which is a time-dependent shear thinning property. Under static conditions, a given fluid will flow (become thin and less viscous) over time when it is shaken, agitated, or otherwise stressed (timedependent viscosity). It then takes a fixed time to return to a more viscous state. Some non-Newtonian pseudoplastic fluids show a time-dependent change in viscosity. The longer the fluid undergoes shear stress, the lower its viscosity. A thixotropic fluid is a fluid that requires a finite time to attain equilibrium viscosity when introduced to a step change in shear rate. When the CM mixes with the blood, the viscosity of CM attempts to establish equilibrium with the blood viscosity, but as long as it is in motion, the CM tends to be less viscous than blood.

\section{Statement 5}

Renal function (SCr levels or GFR) should be assessed in all patients with cancer before the administration of CM to define the best strategy to prevent AKI.

Precision medicine and personalized risk assessments should be implemented, particularly in patients with cancer. Based on the number of risk factors in this specific population, our recommendations are designed for a pragmatic approach that can be easily applied to patients in routine clinical practice. Therefore, we felt comfortable distinguishing 3 different clinical scenarios:

Ambulatory patients: In ambulatory patients, renal function should be assessed by measuring SCr levels (preferably enzymatic method) and calculating the enhanced GFR (eGFR; Table 3 ) within the 2 weeks prior to the CECT.

When a patient with one of the following characteristics (i.e., renal cancer, extensive liver parenchymal involvement, multiple myeloma, or antineoplastic drug [bevacizumab and irinotecan] administration within the $<45$ days prior to CM injection) presents for treatment, the oncologist should obtain enzymatic creatinine measurements and calculate the eGFR within one week of the CECT.

Hospitalized patients: In hospitalized patients, renal function should be assessed by measuring SCr levels (preferably using an enzymatic method) and the GFR on the day before the examination. Patients with a reduced eGFR and clinical manifestations of CKD should be considered at high risk of developing CI-AKI (in this case, acute or chronic). Even in the absence of clinically evident $\mathrm{CKD}$, oncology patients should be considered at a higher risk of developing CI-AKI, particularly elderly patients.

Ideally, after CM administration, patients should be followed using renal function assessments within $48-72 \mathrm{~h}$ of CM administration.
Del Mastro/Laghi/Ronco 
Table 3. Definition of GFR and eGFR

GFR

The GFR describes the flow rate of filtered fluid through the kidney. The creatinine clearance rate $(\mathrm{CCr}$ or $\mathrm{CrCl})$ is the volume of blood plasma that is cleared of creatinine per unit of time and is a useful measure for approximating the GFR.

The GFR is equal to the clearance rate when any solute is freely filtered and is neither reabsorbed nor secreted by the kidneys. Therefore, the measured rate is the quantity of the substance in the urine that originated from a calculable volume of blood. Relating this principle to the equation listed below, for the substance used, the product of the urine concentration and urine flow equals the mass of the substance excreted during the time in which the urine has been collected. This mass equals the mass filtered at the glomerulus because nothing is added in or removed from the nephron. Dividing this mass by the plasma concentration provides the volume of plasma through which the mass must have originated, and thus the volume of plasma fluid that has entered Bowman's capsule within the aforementioned period. The GFR is typically recorded in units of volume per time, for example, millilitres per minute $\mathrm{mL} / \mathrm{min}$ and compared to the filtration fraction.

GFR $=$ urine concentration $\times$ urine flow/plasma concentration.

eGFR

The eGFR is a calculation based on the results of a blood creatinine test and other variables, such as age, sex, and race (e.g., African-American, non-African American), depending on the equation used.

It is used to screen for and detect early kidney damage, to help diagnose CKD, and to monitor kidney status.

The National Kidney Disease Education Program, American Society of Nephrology, and the National Kidney Foundation all recommend that an eGFR be calculated each time a creatinine blood test is performed. The creatinine test is frequently requested as part of a routine comprehensive metabolic panel (CMP), a basic metabolic panel (BMP), or along with a blood urea nitrogen (BUN) test to evaluate the status of a person's kidneys.

Creatinine, along with eGFR, is often used to monitor people with known CKD and people with conditions such as diabetes and high blood pressure (hypertension) that may lead to kidney damage.

In a research setting, additional assessments of renal function in patients at higher risk who have normal baseline SCr levels can be performed using the Kidney Stress Test and by measuring the Renal Functional reserve. In patients undergoing chemotherapy or patients with other conditions associated with higher risks, the measurement of damaged biomarkers may contribute to assessments of ongoing kidney injury and thus the imaging examination should be postponed or re-considered.

\section{Statement 6}

The combination of markers of functional damage (elevation in SCr levels) and structural damage (biomarkers) guarantees more accurate identification of patients with subclinical AKI or those at a higher risk of developing persistent kidney damage.

Elevated SCr levels are a delayed reflection of changes in the GFR, and levels may not peak until 2-3 days after an insult [33]. Furthermore, SCr levels may be affected by several non-renal factors, such as patient characteristics (including muscle mass), drug therapies, fluid overload and laboratory conditions [34]. A growing concern is that the GFR may be sustained even over the course of injuries, since kidneys compensate for damage until approximately $50 \%$ of nephrons are lost. The clinical significance of changes in SCr levels and GFR de-

CT-Related Risk Factors in Oncology Patients pends on patients' underlying renal function to a large extent. The same degree of kidney injury will have different implications for a patient with CKD compared to someone with a high baseline GFR, in whom the injury may produce no clinical symptoms [35]. To ensure that AKI is identified before the damage is severe enough to affect kidney function, alternative or additional measures of kidney injury are needed. Biomarkers of structural damage may help facilitate early AKI diagnosis and treatment initiation. Advances in this field have been made with the discovery and validation of cell-cycle arrest biomarkers, such as tissue inhibitor of metalloproteinase 2 and insulin-like growth factor binding protein 7 [36]. The combination of these biomarkers, which was measured by the approved NephroCheck test [36], appears to be highly predictive of the risk of moderate to severe AKI in the subsequent 24-48 h [37]. However, the NephroCheck test has not yet been validated as a prognostic test for CI-AKI. Several other biomarkers of kidney damage have also been characterized, including neutrophil gelatinase-associated lipocalin [38], kidney injury molecule 1 [39], and N-terminal pro-B-type natriuretic peptide [40]. Of these biomarkers, neutrophil gelatinase-associated lipocalin has shown the most promise as a diagnostic and prognostic tool for AKI. Biomarkers may also have a role in identifying patients at higher risk of enduring kidney damage or relapsing 
Table 4. Estimated risk of developing radiation-related cancer from repeat screens. From Lin et al. [49]

\begin{tabular}{|c|c|c|c|c|}
\hline Study & Screening test & Frequency & Age, years & $\begin{array}{l}\text { Radiation-related } \\
\text { cancers (per 100,000 } \\
\text { screened) }\end{array}$ \\
\hline Kim et al. [75] & Coronary artery calcification CT & Annual & $\begin{array}{l}45-70 \text { (males) } \\
55-70 \text { (females) }\end{array}$ & $\begin{array}{l}40 \text { (males) } \\
60 \text { (females) }\end{array}$ \\
\hline Yaffe and Mainprize [78] & Mammography & $\begin{array}{l}\text { Annual at age }<55 \text { years } \\
\text { Biannual at age }>55 \text { years }\end{array}$ & $45-74$ & 90 (females) \\
\hline
\end{tabular}

kidney dysfunction, for whom the prognosis is poor compared with that of patients who experience early reversal and recovery from kidney injury.

We anticipate that a combination of markers of functional damage (elevated SCr levels) and structural damage (biomarkers) will provide clinicians the greatest opportunity to identify patients with subclinical AKI or patients with the highest risks of developing persistent kidney damage, which would allow for prompt intervention and help preserve patient well-being over the long term. This combined approach would allow patients with either structural or functional damage alone and patients with both structural and functional damage to be identified [41].

\section{Statement 7}

The risk of malignancy from diagnostic radiation exposure in adults is generally low. The uncertainty surrounding this issue should prompt the use of low-dose protocols and adequate technology. Clinicians should take precautions with patients who have been exposed to $10 \mathrm{mSv}$ for a year because these patients have an increased risk of developing new forms of cancer.

Although the individual risk estimated for radiation exposure from medical imaging is small, the concern over CT risks is related to the current rapid increase in CT usage. Small individual risks applied to an increasingly large population may result in a potential public health issue in the future.

Linet et al. [42] estimated that 29,000 future cancers will result from the 70 million CT scans performed in the United States in 2007 . Approximately $0.6 \%$ of the cumulative risk of cancer in a UK population up to 75 years of age is estimated to be attributed to diagnostic X-rays; the corresponding estimates in the United States and Japan are 0.9 and $3 \%$ respectively [43-46]. Moreover, approxi- mately $2 \%$ of the 1.4 million cancers diagnosed annually in the United States may eventually be related to CT scans $[42,47,48]$.

This effect is typically thought be stochastic and can occur at any level of radiation exposure, with the likelihood increasing as the dose increases. The typical lag period between radiation exposure and cancer diagnosis is at least 5 years, and in most cases, the lag may be 1 or 2 decades or longer.

Several evidences reported a definitive correlation between radiation induced cancer risk and dosage greater than $100 \mathrm{mSv}$, not common in medical imaging considering a single examination but frequently reached over a short time period in patients who undergo multiple CT examinations (e.g., CT in emergency setting, evaluation of complications after surgery, follow up for cancer patients) or complex interventional radiology and cardiology procedures using fluoroscopy.

A single CT examination of the abdomen and pelvis usually falls into a radiation dose range of approximately $10 \mathrm{mSv}$. At this dose level, the radiation-induced risks are controversial and no relevant evidences are published at support. According to epidemiological data, an increased cancer risk is observed in the $10-100 \mathrm{mSv}$ range, which is relevant to nuclear cardiac studies and many CT studies [49] (Table 4).

No data support an increased cancer risk at doses less than $10 \mathrm{mSv}$, which is the dose range relevant to radiography and some nuclear medicine and CT studies [49].

\section{Statement 8}

CT protocols should be personalized according to the patient, tumor characteristics and needs to minimize radiation exposure and the amount of CM while maintaining adequate image quality. CT-specific protocols (num- 
Table 5. CT acquisition protocols for the most common clinical scenarios in oncology patients

\begin{tabular}{|c|c|c|c|c|c|c|}
\hline Initial staging & $x^{\complement}$ & $\mathrm{x}$ & $\mathrm{x}$ & $\mathrm{x}^{\pi}$ & $\mathrm{x}^{\Delta}$ & $x^{\circledR}$ \\
\hline Long-term follow-up & $x^{\complement}$ & $x^{€}$ & $\mathrm{x}$ & $x^{\pi}$ & & \\
\hline $\begin{array}{l}\text { ॠ If needed after the arterial and } p \\
{ }^{£} \text { Only in patients with a hypervas } \\
{ }^{\Delta} \text { If peritoneal involvement is sus } \\
{ }^{\circledR} \text { In patients with renal, ureteral } \\
{ }^{€} \text { In patients with suspected hype } \\
{ }^{\circledR} \text { Avoidable if dual energy is avai }\end{array}$ & $\begin{array}{l}\text { rtal phase. } \\
\text { ular primary tu } \\
\text { cted. } \\
\text { d bladder lesio } \\
\text { ascular lesions } \\
\text { ble. }\end{array}$ & $\begin{array}{l}\text { Imour (i.e., mela } \\
\text { ns. }\end{array}$ & noma, renal ca & cer, NET, etc.). & & \\
\hline
\end{tabular}

ber of phases acquired and body regions to be scanned), including the adoption of modern techniques for dose reduction (Automatic Dose Modulation and iterative reconstruction algorithms) should be implemented while considering tumour characteristics and different clinical settings, as well as the diagnostic work-up and disease staging, evaluation of the response to treatment and longterm follow-up.

Multidetector CT (MDCT) is the most frequently used imaging method in clinical practice for the identification and characterization of lesions in oncology patients [50, 51]. Currently, MDCT is considered a real volumetric technique with a spatial resolution of approximately 0.6 $\mathrm{mm}$ [52]. The faster tube rotation (less than $0.3 \mathrm{~s}$ ) reduces the scan time and enables CT studies of the abdomen and pelvis within a single breath-hold. However, the increased number of slices, the submillimetre collimation and the use of multiple dynamic post-contrast phases in a single examination may all contribute to increased radiation exposure. Novel advances in dose reduction methods (e.g., Automatic Dose Modulation systems and iterative techniques for image reconstruction) are routinely implemented in modern scanners and have dramatically decreased radiation exposure. Despite these improvements, the scanning parameters and the number of phases acquired should be chosen carefully according to the clinical scenario.

Theoretically, a complete liver examination might include a pre-contrast scan, followed by a contrast-enhanced acquisition obtained during the arterial (sometimes split into early and late arterial phases), portal venous, and equilibrium phases. For several reasons, including radiation exposure, data explosion with complex image viewing and storage, all 5 phases should not

CT-Related Risk Factors in Oncology Patients be acquired in each patient. Because solid evidence for this issue is not available, the selection and combination of acquisition phases depend on both the clinical questions and the radiologist's experience. An overview of the most common clinical scenarios and protocols is presented in Table 5.

A completely new concept of CT imaging has been introduced with the advent of Dual Energy CT. Dual Energy CT acquires datasets at different photon energy levels in a single CT acquisition and provides information regarding the material composition of tissues based on differences in photon absorption. Moreover, using a dual energy post-processing software, a multiparametric approach to obtaining CT images is feasible. Virtual noncontrast images avoid the need to acquire a pre-contrast scan, iodine maps improve the detection of small hypervascular foci within a lesion, and monoenergetic levels reduce artefacts and increase conspicuity at low $\mathrm{keV}[53$, 54].

Another very promising acquisition protocol that does not require specific CT scans is the Split-bolus method. This innovative technique has the potential to reduce both the number of phases acquired and radiation exposure by splitting the intravenous CM into 2 or 3 boluses and combining phases in a single scan. According to Scialpi et al. [55], this novel approach is an alternative to triphasic MDCT in the initial staging and follow-up of oncology patients.

\section{Statement 9}

Adequate liver enhancement requires personalization of the amount of CM. The CM dose should be calculated according to $\mathrm{mgI}$ rather than volume. The CM dose should be defined according to total body weight (TBW) and, in 
patients with a $B M I>25$, to lean body weight. A range of 500-600 mgI per $\mathrm{kg} \mathrm{BW}$ is considered adequate for liver enhancement. Adequate enhancement of hypervascular liver lesions requires a minimum iodine delivery rate of 1.2 $\mathrm{gI} / \mathrm{s}$ using a standard acquisition protocol.

Vascular and liver parenchymal enhancements are generally affected by different kinetics. Vascular enhancement is determined by the iodine dose delivered per unit of time (Iodine Delivery Rate [IDR]), whereas parenchymal enhancement is influenced by the total iodine dose, which is strictly related to patient body size. The conspicuity of liver lesions, particularly hypovascular lesions, depends on the degree of liver parenchyma enhancement $[56,57]$ and is directly correlated to the dose administered.

The most solid evidence for this topic is derived from 2 historical studies. As shown in the studies by Bae [52] and Yamashita et al. [58], a dose of 500-600 mgI per kg TBW must be delivered to increase liver parenchymal attenuation during the portal phase of at least of 50-60 $\mathrm{UH}[52,58]$. Currently, these doses are considered the minimum amount required for an accurate examination. The simultaneous occurrence of maximal enhancement of liver parenchyma with minimal enhancement of the underlying hypovascular liver lesions improves detection. However, most recent studies recommend tailoring the amount of $\mathrm{CM}$ according to patients' lean body weight (LBW) $[59,60]$. In particular, Kondo et al. [61] recommend injecting $750 \mathrm{mgI}$ per kg of patients' LBW to maximize the lesion detection rate. The rationale for this novel approach is related to the vascular characteristics of adipose tissue, which is poorly perfused compared to parenchymal organs. Therefore, the computation of LBW using a bioimpedance measuring device or through the James formula would provide a more accurate estimation of the amount of $\mathrm{CM}$ to inject without considering the useless contribution of poorly perfused adipose tissue, particularly in obese patients $[59,62]$.

In the case of hypervascular liver lesions, enhancement is directly proportional to IDR ( $\mathrm{gL} / \mathrm{s})$. Radiologists can easily control the IDR by modifying the injection flow rate according to the iodine concentration of a given $\mathrm{CM}$. As a general rule, if the acquisition of an arterial phase for body imaging is required, the IDR should not be less than $1.2 \mathrm{gL} / \mathrm{s}$, but a better IDR is $1.6 \mathrm{gL} / \mathrm{s}$ since it provides higher conspicuity $[63,64]$.

Another very important aspect to consider is the directly proportional relationship between low $\mathrm{kV}$ acquisition protocol and tissue contrast enhancement. Lowering the tube voltage during the $\mathrm{CT}$ acquisition protocol increases tissue contrast enhancement. Iodine better absorbs X-ray photons at low energy because the X-rays are closer to the iodine k-edge. This principle has been widely used, mainly in CT-angiography, and it has potential advantages in clinical practice to reduce both radiation exposure and the amount of CM while maintaining optimal tissue enhancement. As shown in the study by Bae [52], an increase in concentration by $1 \mathrm{mg}$ of iodine per millilitre yields a contrast enhancement of $41.12 \mathrm{HU}$ at 80 $\mathrm{kVp}, 31.74 \mathrm{HU}$ at $100 \mathrm{kVp}$, and $26.18 \mathrm{HU}$ at $120 \mathrm{kVp}$. Moreover, based on the results of Botsikas et al. [65], the total amount of CM in gI could be reduced by $20 \%$ if low $\mathrm{kVs}$ are used.

\section{Statement 10}

No evidence supports the superiority of a specific CM in patients with cancer. The use of IOCM should be preferred due to its higher safety profile and patient tolerance, particularly in intra-arterial injections. Similar trends, although with weaker evidence, are observed for intravenous injections.

No clinical studies have directly investigated the preferred CM for oncology patients. To the best of our knowledge, none of the previously reported studies have compared the diagnostic accuracy of CT using different specific CM. However, concerning the safety profile, useful information has been obtained from studies that assessed clinical outcomes after an intravenous (i.v.) injection of iodinated CM. The results from several non-randomized trials revealed significant clinical and laboratory improvements using IOCM. In previous clinical studies using intra-arterial administration, IOCM (iodixanol) was associated with less patient discomfort than LOCM [66-70].

Few studies have confirmed advantages of IOCM during i.v. administration. Weiland et al. [71] recently performed a randomized controlled trial aimed at comparing the frequency and intensity of discomfort in patients who received i.v. injections of iodixanol and iopamidol for CECT of the abdomen and pelvis. Their results, which were obtained from approximately 300 patients, support the hypothesis that osmolality may be a key determinant of patient discomfort. Patients receiving iodixanol had significantly lower levels of moderate-to-severe or severe discomfort than patients receiving iopamidol, with heat being the major contributor. However, no significant differences in the incidence of overall or contrast-related adverse events were observed/reported between the 2 groups.
Del Mastro/Laghi/Ronco 
Similar results from a larger sample of patients were obtained by Xiao et al. [72], and confirmed the better safety profile of IOCM. These authors reported a nearly threefold increase in the overall frequency of adverse events using iohexol compared to iodixanol ( 0.9 vs. $2.5 \%$, $p<0.05)$. However, laboratory results did not show significant differences across the study groups.

Gharekhanloo and Torabian [73] conducted a clinical trial to compare allergic adverse effects and contrast enhancement between iodixanol and iopromide. Iodixanol seems to produce significantly greater enhancement of the hepatic, aorta and portal veins than iopromide $(p<$ 0.01 ). Immediate reactions, such as nausea and vomiting, were less frequent in the iodixanol group $(p=0.01)$. Late skin reactions, such as rash, were more frequent in the iodixanol group $(p<0.01)$.

\section{Conclusions}

Based on current evidence, the number of patients whose cancer will be complicated by other chronic diseases is expected to increase over the next few decades. A multidisciplinary approach to these patients will be necessary to evaluate the numerous risk factors that are frequently present in patients with cancer. The statements discussed in this study address the clinical implications of numerous CT examinations, which provide clinicians with important and up-to-date information regarding methods to reduce complications in oncology patients.

\section{Ethics Approval and Consent to Participate}

Not applicable. The present paper is a consensus statements paper based on evidences.

\section{Availability of Data and Material}

All the data supporting our findings are presented within the manuscript and the published articles that are mentioned.

\section{Disclosure Statements}

The authors have no competing interests to disclose.

\section{Authors Contribution/Acknowledgements}

General Electric Healthcare conceived the idea to draft the manuscript. Davide Bellini (MD) had been involved as scientific (medical) writers and methodologist. A.L., L.D.M., and C.R. assisted with the preparation of the manuscript content. Maria Grazia Bitti, Lorenzo Preda, Giuseppina Sallustio, Andrea Veltri, Luca Cancian, Michele Scialpi, Pietro Torricelli, Carlo Neumaier, Orlando Catalano, Stefano Canitano, Gisella Ferretti, Massimo Midiri, Maurizio Gallieni, Laura Cosmai, Roberto Scarpioni, Maddalena Barba, Antonio Avallone and Camillo Porta were involved as multidisciplinary team that discussed and voted the statements during a face-to-face meeting of the entire group. They do not meet the criteria for authorship and are listed as contributors.

All authors and contributors revised the manuscript critically for important intellectual content and re-drafted some sections. The recommendations were discussed and revised by all the authors before inclusion in the final version. All the authors have read and approved the final version of the manuscript, and they agreed to be accountable for all aspects of the work to ensure its accuracy and integrity.

\section{Funding Source}

Prex commissioned these Consensus Statements, which were supported by General Electric Healthcare through unrestricted educational grants. The funding agency had no role in the study design, data collection and analysis, the decision to publish or the preparation of the manuscript.

\section{References}

1 Maddams J, Utley M, Moller H: Projections of cancer prevalence in the United Kingdom, 2010-2040. Br J Cancer 2012;107:1195-1202.

2 American Cancer Society. Cancer Treatment and Survivorship Facts and Figures 20162017. Atlanta: American Cancer Society, 2016.

3 Khwaja A: KDIGO clinical practice guidelines for acute kidney injury. Nephron Clin Pract 2012;120:c179-c184.

4 Salahudeen AK, Doshi SM, Pawar T, Nowshad G, Lahoti A, Shah P: Incidence rate, clinical correlates, and outcomes of AKI in pa- tients admitted to a comprehensive cancer center. Clin J Am Soc Nephrol 2013;8:347354.

5 Christiansen CF, Johansen MB, Langeberg WJ, Fryzek JP, Sorensen HT: Incidence of acute kidney injury in cancer patients: a Danish population-based cohort study. Eur J Intern Med 2011;22:399-406.

6 Selby NM, Crowley L, Fluck RJ, et al: Use of electronic results reporting to diagnose and monitor AKI in hospitalized patients. Clin J Am Soc Nephrol 2012;7:533-540.
7 Chertow GM, Burdick E, Honour M, Bonventre JV, Bates DW: Acute kidney injury, mortality, length of stay, and costs in hospitalized patients. J Am Soc Nephrol 2005;16:3365-3370.

8 Cicin I, Erdogan B, Gulsen E, et al: Incidence of contrast-induced nephropathy in hospitalised patients with cancer. Eur Radiol 2014;24: 184-190.

9 Stacul F, van der Molen AJ, Reimer P, et al Contrast induced nephropathy: updated ESUR Contrast Media Safety Committee guidelines. Eur Radiol 2011;21:2527-2541. 
10 Skinner R, Kaplan R, and Nathan PC: Renal and pulmonary late effects of cancer therapy. Semin Oncol 2013;40:757-773.

11 Sendur MA, Aksoy S, Yaman S, et al: Administration of contrast media just before cisplatin-based chemotherapy increases cisplatininduced nephrotoxicity. J BUON 2013;18: 274-280.

12 Perazella MA: Onco-nephrology: renal toxicities of chemotherapeutic agents. Clin J Am Soc Nephrol 2012;7:1713-1721.

13 ACR Manual on Contrast Media, Version 10.2. Reston, VA, USA: American College of Radiology, 2016.

14 Windecker S, Kolh P, Alfonso F, et al: 2014 ESC/EACTS Guidelines on myocardial revascularization: the Task Force on Myocardial Revascularization of the European Society of Cardiology (ESC) and the European Association for Cardio-Thoracic Surgery (EACTS) Developed with the special contribution of the European Association of Percutaneous Cardiovascular Interventions (EAPCI). Eur Heart J 2014;35:2541-2619.

15 Faculty of Clinical Radiology, Standards for Intravascular Contrast Administration to Adult Patients, ed 3. https://www.rcr.ac.uk/ publication/standards-intravascular-contrast-administration-adult-patients-thirdedition (accessed October 24, 2016).

16 Hong SI, Ahn S, Lee YS, et al: Contrast-induced nephropathy in patients with active cancer undergoing contrast-enhanced computed tomography. Support Care Cancer 2016;24:1011-1017.

17 Davidson C, Stacul F, McCullough PA, et al: Contrast medium use. Am J Cardiol 2006;98: $42 \mathrm{~K}-58 \mathrm{~K}$.

18 McCullough PA, Brown JR: Effects of intraarterial and intravenous Iso-Osmolar contrast medium (Iodixanol) on the risk of contrast-induced acute kidney injury: a meta-analysis. Cardiorenal Med 2011;1:220234.

19 Dong M, Jiao Z, Liu T, et al: Effect of administration route on the renal safety of contrast agents: a meta-analysis of randomized controlled trials. J Nephrol 2012;25:290-301.

20 Sperati F, Terrenato I, Barba M, et al: Isotonic Contrast (Iodixanol) Administration vs. Low Osmolar Contrast (Iopromide) Use: Evaluating Risk of Contrast-Induced Nephropathy (CIN) in Cancer Patients at Very Low Risk. Results from the COMEDIANS Trial. Poster No.: C-0175. Congress: ECR, 2014.

21 Preda L, Agazzi A, Raimondi S, et al: Effect on renal function of an iso-osmolar contrast agent in patients with monoclonal gammopathies. Eur Radiol 2011;21:63-69.

22 Nguyen SA, Suranyi P, Ravenel JG, et al: Isoosmolality versus low-osmolality iodinated contrast medium at intravenous contrast-enhanced CT: effect on kidney function. Radiology, 2008;248:97-105.

23 Wang YC, Tang A, Chang D, et al: Long-term adverse effects of low-osmolar compared with iso-osmolar contrast media after coronary angiography. Am J Cardiol 2016;118:985990.

24 Fihn SD, Gardin JM, Abrams J, et al: 2012 ACCF/AHA/ACP/AATS/PCNA/SCAI/STS Guideline for the diagnosis and management of patients with stable ischemic heart disease: a report of the American College of Cardiology Foundation/American Heart Association Task Force on Practice Guidelines, and the American College of Physicians, American Association for Thoracic Surgery, Preventive Cardiovascular Nurses Association, Society for Cardiovascular Angiography and Interventions, and Society of Thoracic Surgeons. J Am Coll Cardiol 2012;60:e44-e164.

25 McCullough PA, Choi JP, Feghali GA, et al: Contrast-induced acute kidney injury. J Am Coll Cardiol 2016;68:1465-1473.

26 Guitterez NV, Diaz A, Timmis GC, et al: Determinants of serum creatinine trajectory in acute contrast nephropathy. J Interv Cardiol 2002;15:349-354.

27 Tervahartiala $\mathrm{P}$, Kivisaari L, Kivisaari $\mathrm{R}$ Vehmas T, Virtanen I: Structural changes in the renal proximal tubular cells induced by iodinated contrast media. Nephron 1997;76: 96-102.

28 Kell DB: Iron behaving badly: inappropriate iron chelation as a major contributor to the aetiology of vascular and other progressive inflammatory and degenerative diseases. BMC Med Genomics 2009;2:2

29 Heinrich MC, Kuhlmann MK, Grgic A, Heckmann M, Kramann B, Uder M: Cytotoxic effects of ionic high-osmolar, nonionic monomeric, and nonionic iso-osmolar dimeric iodinated contrast media on renal tubular cells in vitro. Radiology 2005;235:843-849.

30 Netti GS, Prattichizzo C, Montemurno E, et al: Exposure to low- vs iso-osmolar contrast agents reduces NADPH-dependent reactive oxygen species generation in a cellular model of renal injury. Free Radic Biol Med 2014;68: $35-42$.

31 Seeliger E, Flemming B, Wronski T et al.: Viscosity of contrast media perturbs renal hemodynamics. J Am Soc Nephrol 2007;18:29122920.

32 Bhandari S, Seth A, Sethi KK, et al: Cardiological Society of India practice guidelines for angiography in patients with renal dysfunction. Indian Heart J 2012;64(suppl 2):S18-S43.

33 Moran SM, Myers DB: Course of acute renal failure studied by a model of creatinine kinetics. Kidney Int 1985;27:928-937.

34 Joffe M, Hsu CY, Feldman HI, et al: Variability of creatinine measurements in clinical laboratories: results from the CRIC study. Am J Nephrol 2010:31:426-434.

35 Forni LG, Chawla L, Ronco C: Precision and improving outcomes in acute kidney injury: Personalizing the approach. J Crit Care 2017; 37:244-245.

36 Kashani K, Al-Khafaji A, Ardiles T, et al: Discovery and validation of cell cycle arrest biomarkers in human acute kidney injury. Crit Care 2013;17:R25.
37 Ronco C: Cell cycle arrest biomarkers: new weapons for a new battle. Blood Purif 2014; 38:I-III.

38 Haase M, Bellomo R, Devarajan P, et al: Accuracy of neutrophil gelatinase-associated lipocalin (NGAL) in diagnosis and prognosis in acute kidney injury: a systematic review and meta-analysis. Am J Kidney Dis 2009;54: 1012-1024.

39 Han WK, Bailly V, Abichandani R et al: Kidney injury molecule-1 (KIM-1): a novel biomarker for human renal proximal tubule injury. Kidney Int 2002;62:237-244.

40 Nowak A, Breidthardt T, Dejung S et al: Natriuretic peptides for early prediction of acute kidney injury in community-acquired pneumonia. Clin Chim Acta 2013;419:67-72.

41 Sharma A, Mucino MJ, Ronco C: Renal functional reserve and renal recovery after acute kidney injury. Nephron Clin Pract 2014;127: 94-100.

42 Linet MS, Slovis TL, Miller DL, et al: Cancer risks associated with external radiation from diagnostic imaging procedures. CA Cancer J Clin 2012;62:75-100.

43 Hall E: Milestones in the Radiation Sciences; in Hall EJ, Giaccia AJ (eds): Radiobiology for the Radiologist. 6th ed. Philadelphia: Lippincott Williams and Wilkins, 2006, pp 1-4.

44 Schauer DA, Linton OW: NCRP Report No 160, Ionizing Radiation Exposure of the Population of the United States, medical exposure - are we doing less with more, and is there a role for health physicists? Health Phys 2009;97:1-5

45 United Nations Scientific Committee on the Effects of Atomic Radiation. Sources and Effects of Ionizing Radiation. New York: United Nations Scientific Committee on the Effects of Atomic Radiation, 2008.

46 Muirhead CR, O’Hagan JA, Haylock RG, et al: Mortality and cancer incidence following occupational radiation exposure: third analysis of the National Registry for Radiation Workers. Br J Cancer 2009;100:206-212.

47 Dauer LT, Brooks AL, Hoel DG, et al: Review and evaluation of updated research on the health effects associated with low-dose ionising radiation. Radiat Prot Dosimetry 2010; 140:103-136.

48 Ronckers CM, Doody MM, Lonstein JE, Stovall M, Land CE: Multiple diagnostic Xrays for spine deformities and risk of breast cancer. Cancer Epidemiol Biomarkers Prev 2008;17:605-613.

49 Lin EC: Radiation risk from medical imaging. Mayo Clin Proc 2010;85:1142-1146; quiz 1146.

50 van Kessel CS, Buckens CF, van den Bosch MA, van Leeuwen MS, van Hillegersberg R, Verkooijen HM: Preoperative imaging of colorectal liver metastases after neoadjuvant chemotherapy: a meta-analysis. Ann Surg Oncol 2012;19:2805-2813

51 Kanematsu M, Kondo H, Goshima S, et al; Imaging liver metastases: review and update. Eur J Radiol 2006;58:217-228. 
52 Bae KT: Intravenous contrast medium administration and scan timing at CT: considerations and approaches. Radiology 2010;256: 32-61.

53 Bellini D, Gupta S, Ramirez-Giraldo JC, et al: Use of a noise optimized monoenergetic algorithm for patient-size independent selection of an optimal energy level during dual-energy CT of the pancreas. J Comput Assist Tomogr 2017;41:39-47.

54 Marin D, Nelson RC, Samei E, et al: Hypervascular liver tumors: low tube voltage, high tube current multidetector CT during late hepatic arterial phase for detection - initial clinical experience. Radiology 2009;251:771-779.

55 Scialpi M, Pierotti L, Gravante S, et al: Splitbolus multidetector-row computed tomography technique for characterization of focal liver lesions in oncologic patients. Iran J Radiol 2016;13:e20143.

56 Awai K, Takada K, Onishi H, Hori S: Aortic and hepatic enhancement and tumor-to-liver contrast: analysis of the effect of different concentrations of contrast material at multi-detector row helical CT. Radiology 2002;224: 757-763.

57 Heiken JP, Brink JA, McClennan BL, Sagel SS, Crowe TM, Gaines MV: Dynamic incremental CT: effect of volume and concentration of contrast material and patient weight on hepatic enhancement. Radiology 1995;195:353-357.

58 Yamashita Y, Komohara Y, Takahashi M, et al: Abdominal helical CT: evaluation of optimal doses of intravenous contrast material - a prospective randomized study. Radiology 2000;216:718-723.

59 Ho LM, Nelson RC, Delong DM, Delong: Determining contrast medium dose and rate on basis of lean body weight: does this strategy improve patient-to-patient uniformity of hepatic enhancement during multi-detector row CT? Radiology 2007;243:431-437.

60 Rengo M, Bellini D, De Cecco CN, et al: The optimal contrast media policy in CT of the liver. Part I: technical notes. Acta Radiol 2011; 52:467-472.
61 Kondo H, Kanematsu M, Goshima S, et al: Aortic and hepatic enhancement at multidetector CT: evaluation of optimal iodine dose determined by lean body weight. Eur J Radiol 2011;80:e273-e277.

62 Awai K, Kanematsu M, Kim T, et al: The optimal body size index with which to determine iodine dose for hepatic dynamic CT: a prospective multicenter study. Radiology 2016; 278:773-781.

63 Yanaga Y, Awai K, Nakaura T, et al: Optimal contrast dose for depiction of hypervascular hepatocellular carcinoma at dynamic CT using 64-MDCT. AJR Am J Roentgenol 2008; 190:1003-1009.

64 Matoba M, Kitadate M, Kondou T, Yokota H, Tonami H: Depiction of hypervascular hepatocellular carcinoma with 64-MDCT: comparison of moderate- and high-concentration contrast material with and without saline flush. AJR Am J Roentgenol 2009;193:738744.

65 Botsikas D, Barnaure I, Terraz S, Becker CD, Kalovidouri A, Montet X: Value of liver computed tomography with iodixanol 270, 80 $\mathrm{kVp}$ and iterative reconstruction. World $\mathrm{J} \mathrm{Ra}-$ diol 2016;8:693-699.

66 Palmers Y, De Greef D, Grynne BH, Smits J, Put E: A double-blind study comparing safety, tolerability and efficacy of iodixanol 320 $\mathrm{mgI} / \mathrm{ml}$ and ioxaglate $320 \mathrm{mgI} / \mathrm{ml}$ in cerebral arteriography. Eur J Radiol 1993;17:203-209.

67 Poirier VC, Monsein LH, Newberry PD, Kreps BJ: Double-blind, randomized comparison of iodixanol 320 and iohexol 300 for cerebral angiography. Invest Radiol 1994; 29(suppl 2):S43-S44.

68 Pugh ND, Sissons GR, Ruttley MS, Berg KJ, Nossen JO, Eide H: Iodixanol in femoral arteriography (phase III): a comparative doubleblind parallel trial between iodixanol and iopromide. Clin Radiol 1993;47:96-99.

69 Verow P, Nossen JO, Sheppick A, Kjaersgaard P: A comparison of iodixanol with iopamidol in aorto-femoral angiography. Br J Radiol 1995;68:973-978.
70 Manke C, Marcus C, Page A, Puey J, Batakis $\mathrm{O}$, Fog A: Pain in femoral arteriography. A double-blind, randomized, clinical study comparing safety and efficacy of the iso-osmolar iodixanol $270 \mathrm{mgI} / \mathrm{ml}$ and the low-osmolar iomeprol $300 \mathrm{mgI} / \mathrm{ml}$ in 9 European centers. Acta Radiol 2003;44:590-596.

71 Weiland FL, Marti-Bonmati L, Lim L, Becker HC: Comparison of patient comfort between iodixanol and iopamidol in contrast-enhanced computed tomography of the abdomen and pelvis: a randomized trial. Acta Radiol 2014;55:715-724.

72 Xiao Y, Zeng G, Liu X, Peng C, Lai C, Zhou P: Safety enhancement in adult body computed tomography scanning: comparison of iodixanol versus iohexol. Springerplus 2016; 5:148.

73 Gharekhanloo F, Torabian S: Comparison of allergic adverse effects and contrast enhancement between iodixanol and iopromide. Iran J Radiol 2012;9:63-66.

74 Brenner DJ: Radiation risks potentially associated with low-dose CT screening of adult smokers for lung cancer. Radiology 2004;231: 440-445.

75 Kim MJ, Park CH, Choi SJ, Hwang KH, Kim HS: Multidetector computed tomography chest examinations with low-kilovoltage protocols in adults: effect on image quality and radiation dose. J Comput Assist Tomogr 2009;33:416-421.

76 Berrington de González A, Kim KP, Knudsen $\mathrm{AB}$, Lansdorp-Vogelaar I, Rutter CM, SmithBindman R, Yee J, Kuntz KM, van Ballegooijen M, Zauber AG, Berg CD: Radiation-related cancer risks from CT colonography screening: a risk-benefit analysis. AJR Am J Roentgenol 2011;196:816-823.

77 Brenner DJ, Elliston CD: Estimated radiation risks potentially associated with full-body CT screening. Radiology 2004;232:735-738.

78 Yaffe MJ, Mainprize JG: Risk of radiation-induced breast cancer from mammographic screening. Radiology 2011;258:98-105. Erratum Radiology 2012;264:306. 